\title{
ON A TYPE OF COMPLETENESS CHARACTERIZING THE GENERAL LAWS FOR SEPARATION OF POINT-PAIRS*
}

\author{
BY \\ C. H. LANGFORD
}

In a forthcoming paper by E. V. Huntington $†$ a number of sets of postulates or determining conditions for the type of order called "separation of point-pairs" have been given. These sets are selected from a list of general properties which characterize reversible order on a closed line, and each of the sets is shown to imply all the others so that the several selections are equivalent. It is to be shown in the present paper that sets of postulates for separation of point-pairs are characterized by a property which is closely analogous to ordinary completeness. A class of propositional functions will be defined, to be called general laws, łto which any member of a set of postulates for this type of order belongs, and it will be shown that such sets are sufficient to determine the truth or falsity of any general law which can be constructed on the base $K, R_{4}$, the base for the set. This is a question of deducibility; one or the other of every pair of mutually contradictory general laws on $K, R_{4}$ must be deducible.

The question of deducibility arises here in the following manner. It seems to be true from inductive considerations that each of these sets is a sufficient characterization of the type of order in question and thus that the theorems which follow from any one of them might be held to be exhaustive of the general properties which are understood to attach to systems involving separation of point-pairs. Any such set might then be taken as a set of defining properties for separation of point-pairs in the sense that any theorem which is commonly understood to hold for this type of order is implied by the postulates and no theorem which is recognized as not belonging to this type of order does follow from the postulates. In this sense

* Presented to the Society, January 1, 1926; received by the editors February 18, 1926.

$\dagger$ See Proceedings of the National Academy of Sciences, vol. 11 (1925), pp. 687-689. Also Bulletin of the American Mathematical Society, vol. 31 (1925), p. 405.

$\ddagger$ Cf. E. V. Huntington, Postulates for abstract geometry, Mathematische Annalen, vol. 73 (1913), p. 528. 
the set might be held to embody a satisfactory analysis of the current notion of reversible order on a closed line. On the other hand such a set might, of course, be said to define "separation of point-pairs" in the sense that the connotation of these words is thereby arbitrarily assigned. Now although the properiies which are assigned by any one of Huntington's sets do characterize the type of order under consideration, it may be asked whether some essential property has not been overlooked in the sense that some theorem properly belonging to such systems is not implied by the postulates. Since the number of theorems which follow from sets of this sort is infinite, it would seem that the only way to answer such a question negatively would be to show that the set is complete. A complete set of postulates is a set which is such that any theorem which can be formulated in terms of the given base for the set is either implied by the postulates or else its contradictory is implied by them, that is, one or the other of every pair of mutually contradictory propositions follows from the set. Alternatively, a set is complete if any other set on the same base which implies it is implied by it, the sets in this instance being presumed to be self-consistent. In the case of sets of this kind no relevant theorem can be independent. But in the case of sets of general laws such as those for separation of point-pairs this property is certainly lacking; no categorical existence conditions are introduced and the cardinality of the system is left wholly undetermined.

It is proposed to show, however, that sets of general laws may be complete in a sense quite analogous to that of ordinary completeness and that any one of Huntington's sets does have this property. Since all of these sets are equivalent any one of them may be used, and for the present purpose the selection of postulates given below is most convenient. The system has for base a class $K$ and a tetradic relation $R(a b c d)$ in terms of which the following properties are assigned.

00 . For every $a, b, c, d$ in $K$, if $a b c d$ is true then $a, b, c, d$ are distinct.

$F$. For every distinct $a, b, c, d$ in $K$, some permutation of $a, b, c, d$ forms a true tetrad.

G. For every distinct $a, b, c, d$ in $K, a b c d$...$b c d a$.

$H$. For every distinct $a, b, c, d$ in $K, a b c d . \supset . \overline{a b d c}$.

$R$. For every distinct $a, b, c, d$ in $K, a b c d . \supset . d c b a$.

10. For every distinct $a, b, c, d, x$ in $K, a b c d$. . axcd or $a b c x$.* $^{*}$

It is to be noted that these propositional forms are all hypothetical in that they do not demand that some set of elements have the properties

*The condition that $a, b, c, d$ be distinct in $G, H, R, 10$ is merely a matter of convenience since the hypotheses cannot be satisfied if they are not distinct. 
given in their conclusions in order that the conditions demanded by the postulates be satisfied, but simply that the occurrence of such properties be contingent on the cardinality of $K$. This is of course true of all universal propositions. There is however a more important property which distinguishes sets like this one from other sets, and in order to bring out this distinction it will be necessary to discuss briefly certain well known types of proposition with regard to what may be termed their degree of quantification. Under degree of quantification we may have propositions which are singly quantified as contrasted with those which are multiply quantified.* To use the simplest illustration, such propositions as "For some $a, b, R a b$ holds" or "For every $a, b, R a b$ holds" are singly-quantified propositions, whereas, "For every $a$ some $b$ is such that $R a b$ " is a doubly quantified proposition. Or again, the proposition "Every element has an immediate successor" which occurs in connection with serial relations, when expanded is seen to be $(a):$. ( $\exists b):(c) . a b \cdot \sim a c \vee \sim c b$, which is a triply quantified proposition. Any proposition which involves the applicatives "some" or "every" is quantified, and every variable constituent of the propositional construct has some applicative which applies to it. The degree of quantification of a proposition is determined by the number of occurrences of the applicatives "some" and "every" in the quantifier of the proposition. A singly quantified proposition may be said to be about elementary propositions: $(x) . \phi x$ is about $\phi a$ and $\phi b$, etc. A doubly quantified proposition is, in this sense, about singly quantified propositions: (x) : ( $\mathrm{H} y) \cdot \phi(x, y)$ is about $(\exists y) . \phi(a, y)$ and $(\exists y) . \phi(b, y)$, etc., and each of these singly quantified propositions is about elementary propositions. A triply quantified proposition is about doubly quantified propositions which are about singly quantified propositions, and so on. $\dagger$ In the current theory each variable is assigned a different scope and a separate applicative attaches to each variable. $f$ Thus the proposition (1) For every $x, y, \phi(x, y)$ would be written (2) For every $x$ every $y$ is such that $\phi(x, y)$, or (3) For every $y$ every $x$ is such that $\phi(x, y)$. (2) and (3) are clearly equivalent to (1) which differs from them in that its quantified constituents have the same scope, while in (2), for example, $x$ has a wider scope than $y$. (2) and (3) are doubly quantified and are reducible to (1) which is singly quantified.

\footnotetext{
*The terms are due to Mr. W. E. Johnson, although his use of them differs slightly from the present one. See Mind, new ser., vol. 17, p. 240 ff.

† Compare a paper Some theorems on deducibility, forthcoming, in the Annals of Mathematics.

$\ddagger$ See, however, Principia Mathematica, 2d edition, vol. 1, pp. xx-xxii.
} 
Whenever two applicatives which are both universal or both particular are juxtaposed it is always possible to reduce the degree of quantification in this way, and it is important for our present purpose to effect this reduction, so that when a proposition is referred to as singly quantified it may have $n$ variable constituents but they must all be affected by the same applicative. The present discussion is to be confined to first-order functions. A first-order function is a function whose values are first-order propositions. A first-order proposition is a proposition which contains variables denoting individuals but does not contain any variable functions. ${ }^{*}$ All of the foregoing postulates are first-order functions.

The point about singly quantified propositions is that when a proposition has the form "For every $a, b, c, \cdots, \phi(a, b, c, \cdots)$ " or "For some $a, b, c, \cdots, \phi(a, b, c, \cdots)$ " it refers separately to subclasses of $n$ eiements, so that if we know the relational structure of any such subclass we know whether the proposition is true or false. Now in the case of such expressions $n$ will be finite, so that the relational structure of the subclass can be given extensionally and determinately, and this is important in connection with the subsequent proofs which are concerned solely with single quantifications.

In all of the postulates of the foregoing set the quantified constituents all have the same scope. These postulates are singly quantified. But in adding further properties to the list in order to determine a particular type of reversible order on a closed line we should certainly require other than singly quantified statements. For example we might wish to assign the property of density, "Any two points of the closed line are separated by some pair of points," and this would have to be written $(a, b):$. (Gc, $d): a, b \in K$. . . $c, d \epsilon K \cdot R a c b d$, which is a doubly quantified postulate, and it is not reducible. In fact it will be shown presently that no further independent singly quantified hypothetical first-order functions can be added to the above list, and this is the sense in which the set is to be shown to be complete. By a general law, as used in the present discussion, we mean, then, any first-order functions which is hypothetical and singly quantified.

There are two ways in which the postulates of the set (00-10) may be classified both of which are important. Postulate 00 differs from the other members of the set in that it is concerned with $R$-tetrads in which not all of the elements are distinct. This is brought out by expressing 00 in the form "For every $a, b, c, d$ in $K$, if $a, b, c, d$ are not all distinct, then $R a b c d$ fails." The other postulates have to do with $R$-tetrads in which all of the elements are

*Cf. Principia Mathematica, 2d edition, vol. 1, p. xxiii. 
distinct. It would of course be possible to divide 00 into three different statements having to do respectively with ordered tetrads of one, two, and three distinct elements, but this is unnecessary in the present instance sinceprecisely the same assertion holds for each of these cases. If we consider the possible values of $R$, they fall into four classes according as the tetrads involve one, two, three, or four distinct elements, and these sets of values are non-overlapping. Consequently the validity-values* assigned to any one of the sets are independent of those assigned to any other, and it follows that postulates which refer to different classes in this respect are wholly independent of one another. Since 00 refers solely to the first three sets of tetrads while $F-10$ refer to the fourth set alone, 00 is wholly detached $\dagger$ from the remaining postulates, and for this reason it may be treated separately. On the other hand, we may classify the members of the set (00-10) according to the number of distinct elements in the sets on whose $R$-structure they place restrictions. 00 places restrictions on the $R$-structure of every set of one, two, or three distinct elements; $F$ places restrictions on the structure of every four distinct elements, and the same is true of $G, H$, and $R ; 10$ restricts the $R$-structure of every set of five distinct elements.

Postulates $F, G, H$, and $R$ are alike in that they are about ordered tetrads of distinct elements and involve reference to just four elements. If we consider any subclass of four elements, it is clear that the relation $R$ may hold or fail independently for each of the twenty-four ordered tetrads which may be formed of these elements. The force of $G$ may then be expressed by writing its conclusion in the form $a b c d \cdot \overline{b c d a}=0$, that is, $a b c d \cdot \overline{b c d a}$ cannot occur. Similarly, the conclusion of $H$ may be written $a b c d \quad a b d c=0$, while that of $R$ will be $a b c d \cdot \overrightarrow{d c b a}=0$. In the case of $F$ we should have to write out all of the twenty-four ordered tetrads and exclude the case in which the relation $R$ fails for all of them. The formulation of $F, G, H$, and $R$ in this way shows that their force is to be found in the possibilities which they exclude. Different permutations of the same set of elements are distinguished relatively to one another and to the permutations of related sets, but in no sense absolutely. Accordingly, if a postulate is satisfied by a given selection of validity-values attaching to the ordered tetrads formed of $a, b, c, d$ then the postulate will be satisfied if any of the

\footnotetext{
*A tetrad $a b c d$ has a positive validity-value if $R(a b c d)$ is true and a negative validity-value if $R(a b c d)$ is false.

$\dagger$ Cf. E. V. Huntington, $A$ new set of postulates for betweenness, these Transactions, vol. 26 (1924), p. 275.
} 
elements $a, b, c, d$ are interchanged. The twenty-four permutations of any four elements in $K$ must be characterized by some distribution of positive and negative validity-values; $H$ demands that no two permutations related as are $a b c d$ and $a b d c$ shall both be positive; $F$ demands that not all permutations fail, while $G$ and $R$ demand that no two permutations related as are $a b c d$ and $b c d a$ or as are $a b c d$ and $d c b a$ shall be positive and negative respectively.

If we consider the different forms which arise by distributing positive and negative validity-values in different ways over the several ordered tetrads formed of four elements, the question arises as to how many of these forms are excluded by $F, G, H$, and $R$. If $F, G, H, R$ should exclude all possible distributions, then they would be inconsistent with the existence of at least four elements in the class $K$. If they should be consistent with the occurrence of more than one type of distribution of validity-values, then further restricting conditions independent of $F, G, H$, and $R$ are possible and $F, G, H, R$ do not select a determinate form for any four elements. If, however, there is only one type of distribution of validity-values for the permutations of four elements in ordered tetrads of distinct elements which satisfies $F, G, H$, and $R$, then any further general law about four elements in ordered tetrads of distinct elements is either redundant with $F, G, H, R$ or else, with its addition, the set implies that there are not at least four elements in $K$.

The consideration that $F, G, H, R$ may exclude all possible forms of distribution of validity-values but one leads to an alternative procedure for formulating postulates by which it is possible to know that a determinate form is selected. Instead of attempting to determine when all forms but one have been excluded we may reverse this procedure and demand that any $n$ elements have a specified form. We may express by $R^{\prime}(a b c d)$ the holdure of $R$ for the eight permutations which can be obtained by a cyclic permutation of abcd and its reverse. $R^{\prime}(a b c d)$ means then $R a b c d \cdot R b c d a \cdot R d c b a$, etc. Also, when for some permutation of the elements $x, y, z, w, R$ holds, we may say that $x, y, z$, and whe the form $R(A B C D)$, which means, then, that $R x y z w$ or $R y z w x$ or, etc. If $x, y, z, w, t$ can be so identified with $A, B$, $C, D, E$ that $R(A B C D) \cdot R(B C D E)$ holds, then $x, y, z, w, t$ may be said to have the form $R(A B C D) \cdot R(B C D E)$. Similarly, if for some permutation of $x, y, z, w, R^{\prime}$ holds, we may say that $x, y, z$, w have the form $R^{\prime}(A B C D)$. Consider the postulate

(1) Any four $K$-elements on tetrads of distinct elements have the form (a) $R^{\prime}(A B C D)$; (b) $R$ fails for all permutations other than those involved in (a). 
This formulation is equivalent to the assertion that all forms of proposition other than this one are false; it ascribes a determinate form to any set of four elements on tetrads of distinct elements. ${ }^{*}$ Given any four $K$ elements $a, b, c, d$, then some permutation of $a, b, c, d$ must have this character; what permutation that is is quite irrelevant and is in fact meaningless except in the case of a particular application. That this postulate determines completely the relational structure of $a, b, c, d$ may be seen as follows. Consider the set of all possible ordered tetrads on $a, b, c, d$ and let a set of validity-values be assigned in accordance with the postulate and regard the result as a compound proposition about the holdure and failure of $R$ for the several ordered tetrads. The statement assigns a determinate validity-value to each tetrad of the set. Now consider any rearrangement of $a, b, c, d$ in this proposition. This will give rise to a proposition which must have the same form as the original one and it too will include all possible permutations of $a, b, c$, and $d$. If in the second proposition the validity-value of some permutation of $a, b, c, d$ differs from the validity-value of this permutation in the first proposition, then the two statements are incompatible. If, however, every permutation has the same validity-value in the two cases, then the second is precisely the same proposition as the first. It follows that no two propositions of this kind can both be true. The postulate therefore exhaustively characterizes, in terms of general law, any four elements on tetrads of distinct elements.

It will be shown that (1) is equivalent to $F, G, H$, and $R$. That $F$, $G, H, R$ imply (1) may be seen as follows. Consider any four elements in $K$. By $F$, some permutation of these elements, say $a b c d$, is such that Rabcd holds. Then by $G, b c d a, c d a b$, dabc hold, and by $R, d c b a, a d c b, b a d c, c b a d$ hold. From the fact that the relation $R$ holds for these eight tetrads it follows by $H$ that $R$ fails for all other permutations. But this gives the form demanded by (1).

That $F, G, H, R$ follow from (1) is seen immediately by noting that any four elements having the form demanded in (1) satisfy $F, G, H$, and $R$, so that if every four elements in $K$ have this form, $F, G, H$, and $R$ must hold. This shows that $F, G, H, R$ are complete within the domain to which they refer directly.

We now proceed to formulate, in terms of the notion of the form of a set of elements, a set of postulates for separation of point-pairs which will be shown to be equivalent to the set $00-10$. The set has for base a class $K$

\footnotetext{
*The complete characterization of a set of $n$ elements by assigning a determinate set of validityvalues is due to H. M. Sheffer. The procedure is applied here to values of functions.
} 
and a tetradic relation $R$ in terms of which the following properties are assigned.

1. Every element in $K$ has the form $\overline{A A A A}$.

2. Every pair of elements in $K$ have the form $\overline{A A A B} \cdot \overline{A A B A}$. $\overline{A B A A} \cdot \overline{B A A A} \cdot \overline{A A B B} \cdot \overline{A B A B} \cdot \overline{B A A B} \cdot \overline{A B B A} \cdot \overline{B A B A} \cdot \overline{B B A A}$. $\overline{A B B B} \cdot \overline{B A B B} \cdot \overline{B B A B} \cdot \overline{B B B A}$.

3. Every three elements in $K$ have the form (a) $\overline{A A B C} \cdot \overline{A A C B}$. $\overline{A B A C} \cdot \overline{A C A B} \cdot \overline{A B C A} \cdot \overline{A C B A} \cdot \overline{B A A C} \cdot \overline{C A A B} \cdot \overline{B A C A} \cdot \overline{C A B A} \cdot \overline{B C A A}$. $\overline{C B A A}$; (b) the tetrads obtained by interchanging $A$ and $B$ in (a) are all negative and those obtained by interchanging $A$ and $C$ in (a) are all negative.

4. Every four elements in $K$ on tetrads of distinct elements have the form (a) $R^{\prime}(A B C D)$, (b) $R$ fails for every other permutation of these variables.

5. Every five elements in $K$ on tetrads of distinct elements have the form (a) $R^{\prime}(A B C D) \cdot R^{\prime}(A B C E) \cdot R^{\prime}(A B D E) \cdot R^{\prime}(A C D E) \cdot R^{\prime}(B C D E)$, (b) $R$ fails for every permutation of any four of the variables $A, B, C, D, E$ which is not asserted in (a).

It is clear that 1,2, and 3 can be compounded into one postulate and that they are together equivalent to 00 . It might seem on casual examination as if 4 follows from 5 and is thus superfluous, and this is indeed the case if there are not just four elements in $K$. But if the set 1-5 is to be equivalent to $00-10$, this case cannot be excluded, so that 4 is necessary. The set $1-5$ is not to be regarded as having any value in use; it is introduced here partly as a step in the proof of the completeness of the general laws 00-10 and partly for its theoretical interest.

Theorem I. 00 implies 1, 2, and 3.

For $\overline{a b c d}$ if $a, b, c, d$ are not all distinct.

Theorem II. $F, G, H$, and $R$ imply 4 .

This theorem has already been proved. III and IV are preliminary to $\mathrm{V}$, and are, of course, to be derived from 00-10.

Theorem III. $a b x c \cdot a b c y . \supset$. abxy if $a, b, c, x, y$ are all in $K$.

By 10, $a b x c \cdot y . \supset: a y x c$ or $a b x y$ and $a b c y \cdot x . \supset$. axcy or $a b c x$. But $a b c x$ and $a b x c$ are incompatible, by $H$. Hence $a x c y ; a y x c . \supset$. yxca . ว. $x c a y$, by $G$. But $a x c y$. . $x c y a$, contrary to $x c a y$. Hence $\overline{a y x c}$, hence $a b x y$. 
THEOREM IV. $a b c x \cdot a b c y .3 . a b x y$ or $a b y x$, if $a, b, c, x, y$ are in $K$ and $x \neq y$.

By $G, a b c x . \supset . b c x a$ and $a b c y . \supset . b c y a ; b c x a \cdot y . \supset . b y x a$ or $b c x y$, and $b c y a \cdot x . \supset$. bxya or $b c y x$, by $10 ; b c y x$ and bcxy are contrary, by $H$; hence byxa or bxya. But byxa.ว. $a b y x$ and bxya.ว. $a b x y$.

Theorem V. $F, G, H, R$, and 10 imply 5.

Consider any five elements in $K$. Any four of these elements must have some permutation, say $a b c d$, such that $a b c d$ holds, by $F$. (1) $a b c d \cdot x$ . . axcd or $a b c x$, by 10. Suppose axcd; axcd.ว. xcda.ว.cdax and $a b c d$. . $b c d a$. . $c d a b$. (2) $c d a x \cdot c d a b$. . . $c d b x$ or $c d x b$, by IV. Suppose $c d b x$. Then we have $a b c d \cdot a x c d \cdot b x c d$. Also, $c d b x \cdot a . \supset$. $c a b x$ or $c d b a$, by 10 , and $a b c d$. . . $b c d a$. ว . $c d a b$, by $G$. But $c d a b$ is contrary to $c d b a$, by $H$. Hence $c a b x$. Also, $c a b x$. . . $a b x c$, by $G$, and $a b x c \cdot a b c d . \supset . a b x d$, by III. We have then $a b x c \cdot a b x d \cdot a b c d \cdot a x c d \cdot b x c d$.

Reverting to the alternative possibility in (2), suppose $c d x b$. We have then $a b c d \cdot a x c d \cdot x b c d$. Moreover, $c d x b \cdot a . \supset$. $c a x b$ or $c d x a$, and $c d x a$ is contrary to $c d a x$. Hence $c a x b$. But $c a x b$. . . $a x b c$, and $a x b c \cdot a x c d$. . . axbd. Hence, we have $a x b c \cdot a x b d \cdot a x c d \cdot a b c d \cdot x b c d$.

Reverting to the alternative in (1), suppose $a b c x$. (3) $a b c x \cdot a b c d$ . . $a b d x$ or $a b x d$. If $a b d x, a b d x \cdot c . \supset . a c d x$ or $a b d c$. But $a b d c$ is contrary to $a b c d$. Hence $a c d x$. Also, $a b c d . \supset . b c d a ; b c d a \cdot x . \supset . b c d x$ or $b x d a ; b x d a$. . $a b x d$, contrary to $a b d x$. Hence, $a b c d \cdot a b c x \cdot a b d x \cdot a c d x$. $b c d x$.

Reverting to the alternative in (3), suppose $a b x d$. We have abcd. $a b c x \cdot a b x d$. And $a b x d \cdot c . \supset$. $a c x d$ or $a b x c$. But $a b x c$ is contrary to $a b c x$, hence $a c x d$. Moreover, $a c x d$. . . cxda and $a b c x$. . . cxab, and $c x d a$. $c x a b . \supset$. $c x d b$, by IV. But $c x d b$. $\supset$. $b c x d$. Hence $a b c x \cdot a b c d \cdot a b x d \cdot a c x d$. $b c x d$.

In any case, then, some permutation of any five distinct elements has this $R$-structure. But $a b c x$. . $R^{\prime}(a b c x)$. Similarly, we have $R^{\prime}(a b c d)$, $R^{\prime}(a b x d), R^{\prime}(a c x d)$, and $R^{\prime}(b c x d)$, and this is the form required in the theorem.

Theorems I, II, and V show that set 1-5 follows from set 00-10. The following theorems establish the converse relation.

Theorem VI. 1, 2, and 3 imply 00.

1,2 , and 3 entail the failure of every ordered tetrad in which the elements are not all distinct and this is precisely the force of 00 . 
TheOREM VII. 4 implies $F$.

For if every four elements in $K$ have the form demanded in 4 then not all permutations fail.

Theorem VIII. 4 implies $G$.

An inspection of the form which any four elements must have in accordance with 4 shows that $a b c d \cdot \overline{b c d a}$ cannot occur. Similarly,

Theorem IX. 4 implies $H$ and $R$.

Theorem X. 5 implies 10.

For 10 requires that if $a b c d$ holds and $x$ belongs to $K$, then $\overline{a x c d}$ and $\overline{a b c x}$ cannot both be true. If 5 holds every set of five distinct elements in $K$ must have the form given in 5 and in this form the combination $a b c d \cdot \overline{a x c d} \cdot \overline{a b c x}$ does not occur.

This establishes the equivalence of sets 1-5 and 00-10, and we are now in a position to consider the question whether further independent general laws can be added to either of these sets, and of course if some law exists which is independent of one set it will be independent of the other also. It is a characteristic of the set 1-5 that each of the postulates is expressed in a completely expanded and extensional form. Postulate 4, for example, demands that every four elements in $K$ shall exhibit the type of distribution of validity-values specified in that postulate, and the form there given includes all possible permutations of four elements in tetrads of distinct elements. Now consider any postulate, say $Y$, which is such that it refers to just four elements on tetrads of distinct elements-postulate $G$, for example. In general such a postulate will be satisfied by a set of four elements having certain distributions of validity-values and will not be satisfied by other distributions. If $Y$ is satisfied by a set of elements having the particular distribution of values demanded by 4 , then since 4 demands that every four elements in $K$ have this form, $Y$ will be implied by 4 as it must hold whenever 4 holds. On the other hand, if $Y$ is not satisfied by a set of elements having the particular form demanded in 4 , then 4 implies that $Y$ is false.

This argument requires a slight modification and extension to include extreme cases. In the first place it may happen that $K$ has less than four elements. In this case every general law of the kind is true, and 4 materially implies $Y$. Properly, of course, that $K$ does not have at least four elements implies $Y$; and it may be well to obviate a possible source of confusion 
here. When it is asserted that for every general law $Y, 4$ implies $Y$ or else 4 implies that $Y$ is false, this assertion is not to be understood to mean that for any $Y, 4$ implies that $Y$ is true for every value of $K$ or else 4 implies that $Y$ is false for every value of $K$. It is to be understood to mean that for any value of $K, 4$ implies that $Y$ is true or 4 implies that $Y$ is false. and this again is, of course, to be distinguished from the triviality that for any value of $K$, every $Y$ is such that 4 implies that $Y$ is true or $Y$ is false. For example, the general law $a b c d$. $\supset$. abdc for every $a, b, c, d$ in $K$ is such that if $K$ has at least four elements, Postulate 4 implies that it is false; whereas, if $K$ does not have at least four elements 4 implies that it is true.

There is another special case which needs to be considered. It was said that in general $Y$ will be satisfied by some distributions of validity-values on four elements and will not be satisfied by others. It may happen that $Y$ is satisfied by every distribution of validity-values. In this case, however, $Y$ follows from every general law and itself contributes no postulational information. On the other hand $Y$ may be such that it is incompatible with the existence of at least four elements in $K$. Here, however, if $K$ does have at least four elements then $Y$ must be false and if $K$ does not have at least four elements $Y$ is true. Hence,

THEOREM XI. Every general law $Y$ on the base $K, R$ which refers to just four elements in tetrads of distinct elements is such that 4 implies $Y$ or else such that 4 implies that $Y$ is false.

Precisely similar considerations establish the corresponding theorem with respect to Postulate 5 .

Theorem XII. Every general law $Y$ on the base $K, R$ which refers to just five elements on tetrads of distinct elements is such that 5 implies $Y$ or such that 5 implies the contradictory of $Y$.

Analogous theorems clearly follow for Postulates 1, 2, and 3. But it is also easy to see that here the theorems may be generalized immediately so as to include general laws referring to any finite number of elements. Postulate 1 demands that every element in $K$ have the form $\overline{A A A A}$, and it follows from this that any $n$ elements on tetrads of a single element have a determinate form. Hence any general law referring to tetrads of a single element which is such that it implies that at least one tetrad holds is incompatible with 1 , whereas any such law which does not imply the holdure of at least one tetrad aaaa is implied by 1 . Similar considerations hold for 2 and 3; 2 implies that for any $n$ elements on ordered tetrads of two 
distinct elements every tetrad fails, and 3 implies that any $n$ elements on tetrads of three distinct elements are such that every tetrad fails. Hence,

THEOREM XIII. Every general law on the base $K, R$ which involves reference to $n$ elements on ordered tetrads of elements not all distinct is such that it is implied by Postulates 1, 2, and 3 or else such that its contradictory is implied by 1, 2, and 3.

It is not always the case that a postulate refers solely to tetrads of nondistinct elements or solely to tetrads of distinct elements; many restrict in some way the validity-values of both. In dealing with such a possibility it is necessary to consider the combined force of Theorems XI, XII, and XIII, and indeed we have already combined 1, 2, and 3 additively in arriving at XIII. Consider any four elements in $K$ formed into all possible ordered tetrads of distinct and non-distinct elements. XIII requires that $R$ fail for all tetrads of non-distinct elements, whereas XI prescribes a determinate form for those tetrads formed of distinct elements. Clearly, the total form exhibited by any four elements is a determinate one, and any general law whatever, a value of which involves not more than four elements, is dependent on XI and XIII. Similarly, XII and XIII imply that any five elements in $K$ have a completely expanded form with a determinate distribution of validity-values. Hence,

TheOREM XIV. Any general law which can be formulated in terms of $K$ and $R$ and whose values do not involve more than five elements is such that either it or its contradictory is implied by the set 1-5.

It is certain, then, that if there exist any general laws independent of 1-5 they must have values which involve more than five elements. In order to show that such laws do not exist it will be necessary to show that as a consequence of 1-5 any $n$ elements have a determinate form. Postulates 1,2 , and 3 have already been shown to include the case of $n$ elements where $n$ is any finite number. It remains to extend 5 in this way.

We may denote by $C(a b c \cdots)$ the assertion of all those ordered tetrads which are obtained in the expression $C$ by reading in the order from left to right. Thus $C(a b c d e)$ means $R a b c d \cdot R a b c e \cdot R b c d e$, etc.

THEOREM XV. For every set of $n$ elements in $K$ ( $n>3$ and finite) some permutation $a b c \cdots$ is such that $C(a b c \cdots)$ holds.

The theorem has already been established for $n \leqq 5$ in XI and XII. Consider the hypothesis that the theorem holds for every subclass in $K$ of just $n$ elements, where $n \geqq 5$. 
Any subclass of $K$ having $n+1$ elements has $n+1$ subclasses of $n$ elements each, and for each such subclass the relation $C$ holds for some permutation of its elements.

It will be shown that there are at least three elements $a, b, c$ in the set of $n+1$ elements such that $a b c x$ holds for every element $x$ of the set other than $a, b$, and $c$.

Take any subclass of $n$ elements. Some permutation $C(a b c \cdots)$ holds. Call the element not belonging to this set of $n$ elements $q$. Now $a b c x$ holds for every $x$ other than $a, b$, and $c$ in the expression $C$. If $a b c q$, then the requirement is satisfied.

Let $z$ be some one of the set of $n+1$ elements other than $a, b, c$, and $q$. Then $a b c z$ holds. By 10, $a b c z \cdot q . \supset . a b c q$ or $a q c z$. Suppose $a q c z$. We have $a b c z ; a b c z . \supset . b c z a . \supset . c z a b ; c z a b \cdot q . \supset . c q a b$ or $c z a q$. If $c q a b$, then $a b c q$, and the requirement is satisfied. If the condition is not to be satisfied $c z a q$ must be true. $c z a q$. . $q a z c . \supset . a z c q$, and $a b c z$. . . $b c z a$. . . $a z c b$; $a z c q \cdot a z c b . \supset$. $a z q b$ or $a z b q$. Moreover, $a z q b . \supset$. $z q b a . \supset$. $a b q z$, and $a z b q$ . ว . zbqa . . . aqbz.

Suppose $a b q z$. Then $a b q z$. . zqba . . $q b a z$. . . $b a z q$, and $a b c z$. . $z c b a$ . د . cbaz . . . bazc; bazq b bazc . . . baqc or bacq.

If $b a q c, b a q c$. . . abcq. Here $a, b$, and $c$ satisfy the condition.

If $b a c q, b a c q . \supset . a b q c$, and $a b q c \cdot a b c x . \supset . a b q x$. Here $a, b$, and $q$ satisfy the condition.

Suppose $a q b z$. Then $a q b z \cdot c$. . . $a c b z$ or $a q b c$. But $a b c z . \supset$. zabc and $a c b z .3 . z a c b$, contrary to $z a b c$. Hence $a q b c$. Now $a q b c \cdot x . \supset$. $a x b c$ or $a q b x$. But $a x b c$ is contrary to $x a b c$. Hence $a q b x$. Here $a, q$, and $b$ satisfy the condition. Hence, for some $a, b, c, a b c x$ for every $x$ other than $a, b$, and $c$ in the set of $n+1$ elements.

Let $a, b, c$ be three elements in the set such that $a b c x$ for every $x$ other than $a, b, c$, and let the relation $C$ hold for the set of $n$ elements to which $c$ does not belong. $C(x y z \cdots w t)$. ว. $C(t w \cdots z y x)$, so that for one or the other of these expressions $a$ and $b$ occur in the order $a b$. Consider the expression for which this is the case.

There is no pair of elements $x, y$ in $C$ such that $a x b y$. For $a b c x \cdot a b c y$ . כ.abxy or $a b y x$. If $a b x y, a b x y$. . $y a b x$ and $a x b y$. . yaxb, contrary to $y a b x$. If $a b y x, a b y x . \supset . x y b a . \supset . y b a x$ and $a x b y . \supset . y b x a$, contrary to $y b a x$. Then $a x b y$ is always false. Hence, in the expression $C$ every element $x$ is such that $a, x$, and $b$ are in the order $a x b$ or no element is so.

We may permute the elements of $C$ cyclically so as to bring $a$ into the first place. We have then either $C(a \cdots x \cdots b)$ or $C(a b \cdots x \cdots)$. If $C(a \cdots x \cdots b)$, then $C(\cdots x \cdots b a)$ and this implies $C(a b \cdots x \cdots)$; 
so that in any case $C$ can be so chosen that $a$ and $b$ fall in the first and second places respectively. We have then $a b x y$ for every $x, y$ in the order $x y$ in $C$. $a b c x$. . . $x c b a$. . . $c b a x$ and $a b c y$. . . ycba . . . cbay; cbax . cbay . . . cbxy

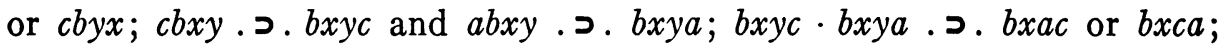
$b x a c$. . . $x a c b$, contrary to $x a h c$, and $b x c a$. . . $a b x c$, contrary to $a b c x$. Hence $\overline{c b x y}$, hence $c b y x$; but $c b y x$. . . $x y b c$. . . $b c x y$.

$a b x y$. . $y x b a$ and $b c x y$.د. $y x c b ; y x c b \cdot y x b a$. . . yxca; $y x c a$.د. $a c x y$. So that we have $a b c x \cdot a b c y \cdot a b x y \cdot a c x y \cdot b c x y$. We have also $a x y z$ and $b x y z$, reading from left to right in $C$. It remains to be shown that $c x y z$ holds.

$b c x y$. د. $c x y b ; c x y b \cdot z$. . . $c x y z$ or $c z y b ; c z y b$. . . $b c z y$, contrary to bcyz. Hence $c x y z$ if $a x y z$ and $b x y z$, and we have $C(a b c \cdots x y z)$ for some permutation of the set of $n+1$ elements, which is the theorem.

If $R a b c d$ holds, then $R b c d a$ and $R d c b a$. It follows that $R$ holds for every tetrad which can be obtained by a cyclic permutation and its reverse. We may express, as previously, the holdure of $R$ for these eight permutations by writing $R^{\prime}(a b c d)$. We may express by $C^{\prime}(a b c \cdots)$ the assertion that $R^{\prime}$ holds for any four elements in $C^{\prime}$ which occur in the order from left to right.

THEOREM XVI. Any $n$ elements in $K$ are such that for some permutation $a b c \cdots, C^{\prime}(a b c \cdots)$ holds and such that $R$ fails for every other permutation of any four of these elements.

$C(a \dot{u} c \cdots)$ holds by XV. But Rabcd. $\supset . R^{\prime}(a b c d)$. Hence $C^{\prime}(a b c \cdots)$. That all other ordered tetrads fail follows from Postulate 4.

We have then the following exhaustive characterization of any set of $n$ elements in $K$.

(a) For some permutation $C^{\prime}(a b c \cdots)$ holds where $a, b, \cdots$ are all distinct.

(b) For every ordered tetrad of distinct elements which does not occur in $C^{\prime}, R$ fails.

(c) For every ordered tetrad of the elements in $C^{\prime}$ which are not all distinct $R$ fails.

(c) follows from postulate 00. If $n$ is less than four (c) alone is relevant. It is clear that in view of (a), (b), and (c), any $n$ elements in $K$ have a determinate form; no further independent general laws can be added to the set $00-10$.

All of the general laws $00-10$ are universal in that they impose the same condition on every set of $n$ elements in $K$ and they are all hypothetical in that they are satisfied if $K$ does not have at least $n$ elements. We may, 
however, have general laws which are not universal, though they must of course be hypothetical. Such laws are of the form "If there are at least $n$ elements in $K$, then for some $a, b, c, \cdots, n, \phi(a b c \cdots n) . "$ And it is clear that a universal law implies the corresponding hypothetical particular, so that each member of the set 00-10 implies a corresponding particular function. Thus "For every distinct $a, b, c, d$ in $K, a b c d$. $\supset . d c b a$ " implies that if there are at least four elements in $K$, then for some $a, b, c, d$ in $K$, $a b c d . \supset . d c b a$; "For every distinct $a, b, c, d, x$ in $K, a b c d$. . . axcd or $a b c x$ " implies that if $K$ has at least five elements, then for some $a, b, c, d, x$, $a b c d . \supset$. axcd or $a b c x .^{*}$ Conversely, any property which can be assigned by a singly quantified first-order function and which belongs to at least one set of $n$ elements in $K$ belongs to every such set.

It is a characteristic of the set $00-10$ that the holdure or failure of the relation $R$ is left wholly undetermined for any tetrad $a b c d$ whose elements do not all belong to the class $K$. The foregoing theorems have been concerned solely with the determination of the form of any set of $n$ elements all of which belong to $K$, and it is with respect to these elements that the set is complete as to general law. It seems to be commonly assumed, with regard to sets of this kind, that if a set of say four elements in the case of a tetradic $R$ does not fall wholly within $K$, then $R$ fails. It is sometimes suggested that $R$ is undefined for such sets of elements. This can, however, mean no more than that $R$ fails universally in such cases, for when we write "If $a, b, c, d$ belong to $K$, then $\phi(a, b, c, d)$ " it is presupposed that $\phi(a, b, c, d)$ is significant even if $a, b, c, d$ do not all belong to $K$. We may then subjoin to the set 00-10 the condition that if $a, b, c, d$ do not all belong to $K$, then Rabcd fails. With this condition any set of $n$ elements within the range of significance of the variables $x, y$ in $R x y$ has a unique $R$-structure with respect to any general law.

- Cf. Huntington, Proceedings of the National Academy of Sciences, loc. cit., p. 688.

HARVARD UNIVERSITY,

Caymrimge, Mass. 